\title{
Malformación linfática facial mixta. Caso clínico
}

\author{
C. G. Morovic • C. Vidal • N. Leiva ・ S. Véliz
}

\begin{abstract}
Mixed facial lymphatic malformation - Case report

Lymphangiomas are a common form of vascular malformation of the lymphatic vessels, mainly in the head and neck region. Most cases are progressive evolution and require a multidisciplinary approach. Currently, the first therapeutic option is sclerotherapy, leaving surgery for the treatment of remaining lesions. Objective: To present a case of facial lymphatic malformation (LM) treated with sclerotherapy, surgery and orthodontics in a 15-year follow up. Case report: A one-year-old female patient who consulted health professionals due to a progressive volume increase of the soft parts of her right cheek. The imaging study confirmed the diagnosis of microcystic lymphatic malformation. It was managed with OK-432 sclerotherapy and Bleomycin. At 2 years of age, the patient response was considered adequate; an intralesional submandibular surgical excision was then performed, with partial resection of the lesion. The biopsy confirmed the diagnosis of microcystic LM. Six months after, a re-resection was planned using the same approach and removing the remaining lesion, with favorable development until the age of 9 years when the patient required surgery and orthodontic management due to intraoral recurrence. No major developments until the age of 13 when a new orthodontic surgery and handling are planned to perform right oral commissure suspension. Conclusion: LM management by sclerotherapy, surgery, and orthodontics has shown the advantages of a multidisciplinary long-term treatment in this case.
\end{abstract}

(Key words: Lymphangioma, lymphatic malformation, lymphatic vessel tumors, sclerotherapy).

Rev Chil Pediatr 2014; 85 (6): 714-719

\section{RESUMEN}

El linfangioma corresponde a una malformación vascular de los vasos linfáticos, preferentemente de la región de cabeza y cuello. La mayoría de los casos son de evolución progresiva y requieren un manejo multidisciplinario. Actualmente la primera opción terapéutica es la esclerosis, reservando la cirugía para el tratamiento de las lesiones remanentes. Objetivo: Presentar un caso de malformación linfática (ML) facial, tratado con

Recibido el 27 de marzo de 2014. Última versión aceptada el 09 de noviembre de 2014.

Carmen Gloria Morovic I. $(\bowtie)$

Cirujano plástico pediátrico. Hospital Luis Calvo Mackenna-Clínica Las Condes.

E-mail: cmorovic@gmail.com

Claudia Vidal T.

Cirujano pediátrico. Hospital Luis Calvo Mackenna-Clínica Santa María.

Noemí Leiva V.

Ortodoncista. Facultad de Odontología. Universidad de Chile. Hospital Luis Calvo Mackenna.

Sebastián Véliz M.

Cirujano Dentista. Facultad de Odontología, Universidad de Chile. 
escleroterapia, cirugía y ortodoncia en un seguimiento a 15 años. Caso clínico: Paciente de sexo femenino que consulta al año de edad por aumento de volumen progresivo de partes blandas en su mejilla derecha. El estudio de imágenes confirmó el diagnóstico de Malformación Linfática microquística. Se manejó con esclerosis seriada con OK-432 y Bleomicina. A los 2 años de edad se consideró que la respuesta era adecuada, y se procedió a realizar extirpación quirúrgica intralesional submandibular, con resección parcial de la lesión. La biopsia confirmó el diagnóstico de ML microquística. Seis meses después se planificó nueva resección utilizando el mismo abordaje y extirpando lesión remanente, con evolución favorable hasta la edad de 9 años en que requiere cirugía y manejo por ortodoncia, por recidiva de lesión a nivel intraoral. Evolución favorable hasta que a la edad de 13 años se planifica nueva cirugía y manejo por ortodoncia para suspender la comisura bucal derecha. Conclusión: El manejo de la ML mediante escleroterapia, cirugía, y ortodoncia muestra en este caso las ventajas de un tratamiento multidisciplinarion a largo plazo.

(Palabras clave: Linfagioma, malformación linfática, tumor, vasos linfáticos, escleroterapia).

Rev Chil Pediatr 2014; 85 (6): 714-719

\section{Introducción}

La malformación linfática (ML) corresponde a una anomalía del desarrollo de los vasos linfáticos, los cuales se encuentran dilatados y llenos de linfa, formando quistes de tamaño variable. Representan una de las malformaciones vasculares más frecuentes. El diagnóstico es en base a la sospecha clínica y se confirma por imágenes, las que son bastante características y permiten establecer el tamaño de los quistes ${ }^{1-3}$.

La resonancia nuclear magnética (RNM) es el estudio de elección, especialmente cuando hay compromiso de otras estructuras vasculares, y para establecer la relación con estructuras vecinas, como la compresión de vía aérea.

E1 $80 \%$ de los casos se manifiestan durante los 2 primeros años de vida, cuya sintomatología dependerá de la zona donde se encuentra ya sea deformidad, dificultad respiratoria, anomalías dentomaxilares por invasión del tejido entre las piezas dentarias, dificultad para deglutir y/o hablar, infecciones y/o dolor. $\mathrm{Su}$ localización es variable, predominando la región cérvico-facial $(75 \%)$ y la zona axilar $(20 \%)$. Otras ubicaciones de menor frecuencia son mediastino, retro peritoneo, lengua y extremidades. La regresión espontánea de las ML está descrita hasta el 6\%, dependiendo de las series.

La necesidad de tratarlas esta dado básicamente por su crecimiento progresivo que puede provocar deformidad, compresión de estructuras vecinas y posibilidad de sangramiento y/o infección intralesional.

Originalmente estos pacientes se trataban con exéresis quirúrgica pero esto ha ido cambiando dado las complicaciones, secuelas y altos índices de recurrencia de las lesiones. Por otra parte, los esclerosantes han tomado mayor protagonismo demostrando tener alta eficacia en muchos casos con mínimos efectos colaterales ${ }^{4}$.

Se han utilizado múltiples agentes esclerosantes entre los cuales se encuentra la bleomicina, alcohol, polidocanol, OK-432 entre otros, reportándose diferencias en la respuesta de la ML según las características morfológicas de las lesiones, más que del agente en particular utilizado siendo más exitoso en los macro quísticos y uniquisticos ${ }^{5,6}$.

El objetivo del presente artículo es mostrar un caso de ML facial mixta, en el que se trató multidisciplinariamente con escleroterapia, cirugía y ortodoncia en un seguimiento a 15 años.

\section{Caso clínico}

Paciente que consultó al año de edad por un aumento de volumen progresivo en su mejilla derecha, con asimetría facial, descenso de comisura oral derecha, sin compromiso esqueletal, cuya clínica y radiología era compatible con ML (figura 1A). La ecografía facial, a los 18 meses de edad, realizada con transductor 

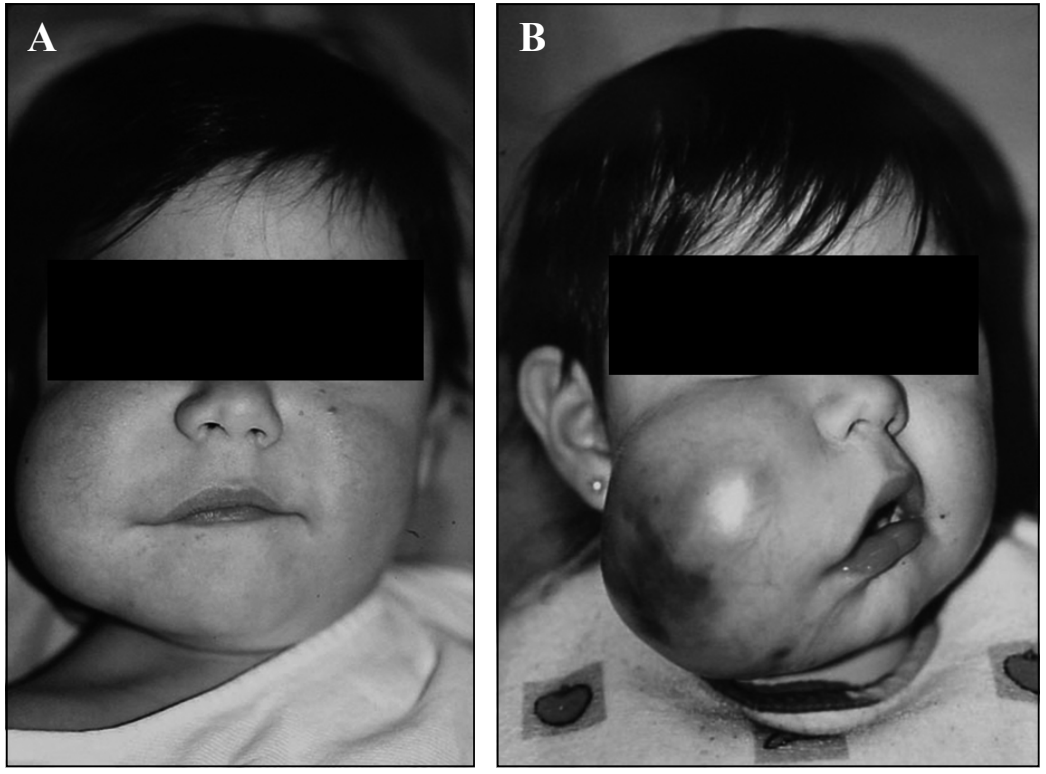

Figura 1. (A) Paciente de 18 meses de edad portadora de ML en mejilla derecha de aumento progresivo; (B) Aspecto clínico post escleroterapia con OK432.

lineal de alta resolución, mostraba lesión predominantemente micro quística, con algunos quistes que superaban los $15 \mathrm{~mm}$ de diámetro.

Se planificó realizar esclerosis seriada de la lesión, para lo cual se utilizó en 2 ocasiones + $\mathrm{OK}-432$ y en las 2 siguientes Bleomicina. Todas se realizaron con 6-8 semanas de intervalo bajo anestesia general. El procedimiento consistió en el aspirado del contenido de los quistes de mayor tamaño e inyección de $\mathrm{OK} 432$, en dosis de $0,1 \mathrm{mg}$ disueltos en $10 \mathrm{cc}$ de volumen total. Durante el primer procedimiento, al $4^{\circ}$ día presentó un importante aumento de volumen e inició un cuadro de escarlatina (figura 1B), evolucionando con una disminución de la proyección de la mejilla e induración local. Similar procedimiento se realizó con la Bleomicina cuyas dosis no sobrepasaban $0,3-1 \mathrm{mg} /$ $\mathrm{kg}$ por sesión. En las posteriores infiltraciones, presentó aumento de volumen más discreto y con menor reacción local.

A la edad de 2 años 4 meses, con la lesión más circunscrita, se consideró que no tenía mayor beneficio continuar con las esclerosis y se planificó extirpación quirúrgica intralesional (figura 2A). Se utilizó abordaje externo submandibular derecho, con resección parcial de la lesión, la que comprometía espesor completo de la mejilla incluyendo musculatura facial y mucosa vestibular. A los 15 días post operatorio se inició tratamiento compresivo elástico y masoterapia, intentándose compresión externa con máscara de acrílico pero que fue mal tolerada por la paciente.

La biopsia confirmó el diagnóstico de ML microquístico con intersticio fibroso e infiltración inflamatoria linfocitaria. Seis meses después se planificó nueva resección utilizando el mismo abordaje y extirpando lesión remanente hacia cefálico (figura 2B). En el post operatorio se mantuvieron las medidas de compresión facial previas a la cirugía.

Después de varios años comenzó a presentar molestias en relación a lesiones vesiculares en vestíbulo oral que se mordía con frecuencia, siendo interpretadas como recidiva local de su ML (figura 2C). A los 9 años de edad, se realizó exéresis de la lesión con abordaje intraoral, cuya biopsia mostraba ML con fibrosis difusa. Dado el difícil manejo de la cicatrización intraoral ya que la paciente se mordía con frecuencia presentando sangramiento e inflamación local, se derivó a ortodoncia para manejo de su anomalía dentomaxilar y confección de un plano de apoyo intraoral.

Se diseñó un aparato intraoral con prolongación vestibular completa que proporcionaba un buen plano de apoyo intraoral que potencia- 

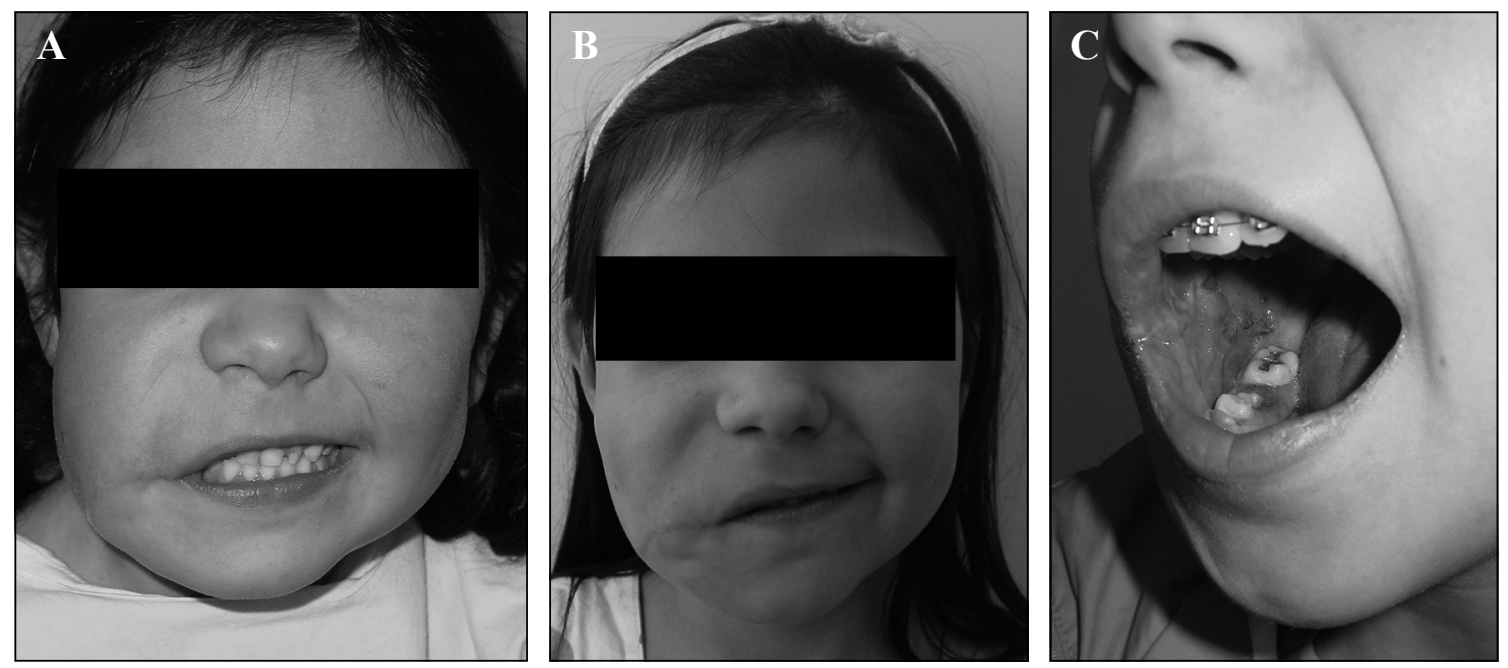

Figura 2. Después de cuatro procedimientos de esclerosis (A) pre operatorio; (B) A los 2 años post cirugía; (C) A los 9 años presenta recidiva en zona vestibular con interposición de mucosa comprometida al masticar.

ba la compresión externa (figura 3, A-B), Además se instaló aparatología ortodóncica fija en la zona dentaria anterosuperior para alinear y nivelar el sector incisivo (figura 3, C-D). La compresión interna/externa se indicó por $15 \mathrm{~h}$ diarias, con controles del aparato y rebasado de acrílico de acuerdo al espacio que se iba produciendo en el vestíbulo.

Dado que el seguimiento de los últimos 4 años no mostró recidiva local y la comisura oral derecha se encontraba descendida e inanimada, se programó una nueva cirugía para suspender la comisura derecha, ayudado por el soporte intraoral al que se le rebasa material semanalmente agregando una zona más alta que levanta y da soporte a la comisura.

\section{Discusión}

La denominación de ML reemplaza a la antigua terminología de linfangioma, linfangioma capilar, higroma quístico, entre otras denominaciones que llevaban a confusión. La clasificación de Mulliken y cols ubica las ML dentro de las malformaciones vasculares que comprometen a los vasos linfáticos ${ }^{7}$. Según sus características morfológicas y/o imágenes radiológicas pueden ser macro quísticos, micro quísticos o mixtos, lo que tiene connotación desde el punto de vista de su tratamiento y pronóstico. Según distintos reportes ${ }^{4}$ los macro quísticos tienen mejor respuesta a los esclerosantes, no así los microquísticos, las malformaciones vasculares mixtas y los pacientes que han tenido cirugías previas.

El OK-432 (PICIBANIL) es una mezcla liofilizada de Streptococo pyogenes del grupo A, tipo 3 de origen humano tratada con Penicilina $\mathrm{G}$ Potásica, que inactiva su virulencia, pero que mantiene su capacidad de generar una reacción inflamatoria local. Al inyectarse OK- 432 genera una reacción inflamatoria local y posterior reducción de la masa tumoral por adhesión y subsecuente fibrosis intraluminal de los vasos ${ }^{8-13}$. Existen reportes referentes a resultado exitoso después de un promedio de 2 infiltraciones en LM macro/uniquisticas y 6 veces para el tipo microquístico ${ }^{14-16}$. Teniendo además como ventaja sobre la cirugía de evitar lesiones vasculares, nerviosas y de estructuras vitales adyacentes.

La bleomicina es un antibiótico antitumoral citotóxico que induce la degradación del ADN y por otra parte genera esclerosis del endotelio vascular. Tiene una amplia disponibilidad, bajo costo y mínimos efectos adversos. Su uso intralesional no provocaría efectos tóxicos sobre médula ósea ni compromiso pulmonar crónico ya que el pasaje a la circulación es muy 

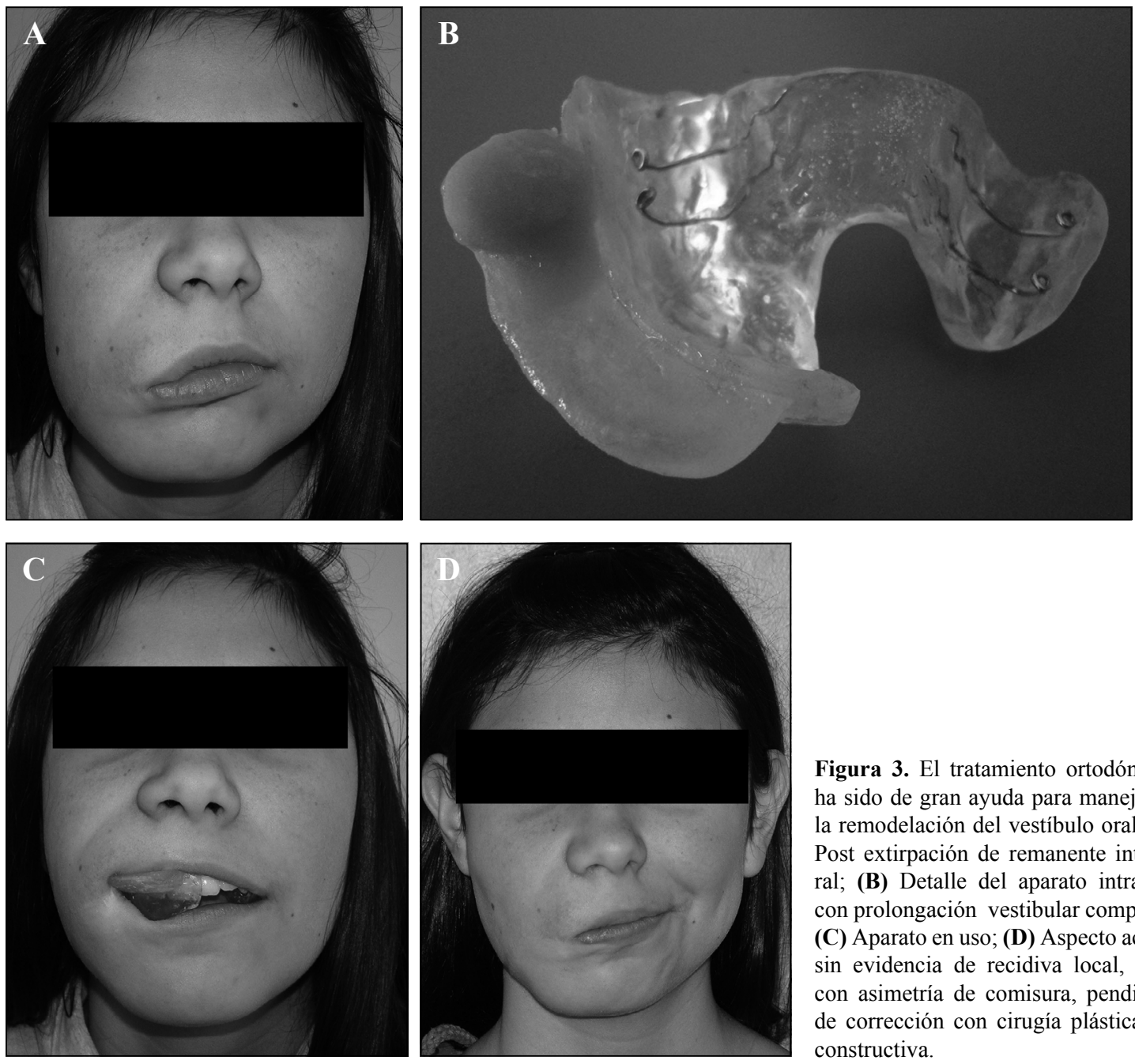

Figura 3. El tratamiento ortodóncico ha sido de gran ayuda para manejo de la remodelación del vestíbulo oral (A) Post extirpación de remanente intraoral; (B) Detalle del aparato intraoral con prolongación vestibular completa; (C) Aparato en uso; (D) Aspecto actual sin evidencia de recidiva local, pero con asimetría de comisura, pendiente de corrección con cirugía plástica reconstructiva.

marginal y las dosis utilizadas no sobrepasan 0,3-1 $\mathrm{mg} / \mathrm{kg}$ por sesión ${ }^{17}$.

Estudios comparativos de ambos esclerosantes no han demostrado diferencias significativas en el éxito del tratamiento. En pacientes con ML micro quístico que serán sometidos a cirugía tendría el beneficio de reducir el tamaño de la lesión y disminución de la linforragia postoperatoria.

La cirugía por muchos años el tratamiento de elección, es compleja, con riesgo de lesión de estructuras nobles comprometidas y muchas veces no logra la extirpación completa de la $\mathrm{ML}^{18}$. Actualmente solo estaría reservada para lesiones pequeñas bien circunscritas, como tratamiento único, extirpación de remanente fibroso previamente esclerosado o reducción intralesional de LM de gran tamaño (en conjunto con esclerosis). La cirugía, presenta tasas más altas de recurrencias, de complicaciones y de enfermedad sintomática persistente.

La recidiva del linfagioma tras el tratamiento con OK-432 según Luzzatto y cols. ${ }^{19-20}$ es del $11 \%$.

En el caso presentado, la terapia esclerosante con OK-432 y bleomicina, disminuyó en forma importante el tamaño de la lesión inicial, facilitando la cirugía posterior. Presenta recurrencia local después de 6 años, realizándose extirpación quirúrgica del remanente y manejo 
ortodóncico para tratamiento de deformidades dentomaxilares y coadyuvante en la compresión elástica y remodelación del vestíbulo oral.

Potenciales conflictos de interés: Este trabajo cumple con los requisitos sobre consentimiento/asentimiento informado, comité de ética, financiamiento, estudios animales y sobre la ausencia de conflictos de intereses según corresponda.

\section{Referencias}

1.- Kennedy TL, Whitaker M, Pellitteri P, Wood WE: Cystic hygroma/lymphangioma: a rational approach to management. Laryngoscope 2001; 111: 1929-37.

2.- Orvidas LT, Kasperbauer JL: Pediatric lymphangiomas of the head and neck. Ann Otol Rhinol Laryngol 2000; 109: 411-21.

3.- Zadvinskis DP, Benson MT, Kerr HH, et al: Congenital malformations of the cervicothoracic lymphatic system: Embryology and pathogenesis. Radiographics 1992; 12: 1175-89.

4.- Sanlialp L, Karnak L, Tanyel FC, et al: Sclerotherapy for Lymphangioma in Children. Int J Pediatr Otorhinolaryngol 2003; 67 (7): 795-800.

5.- Acevedo J, Shah R, Brletzke S: Terapia no quirúrgica de Linfangiomas: Una revisión sistemática. Rev Otorrinolaringol Cir Cabeza Cuello 2008; 68: 323-30.

6.- Burrows P, Mason K: Percutaneous Treatment of Low Flow Vascular Malformations. J Vasc Interv Radiol 2004; 15: 431-45.

7.- Mulliken JB, Glowacki J: Hemangiomas and vascular malformations in infants and children: a classification based on endothelial characteristics. Plast Reconstr Surg 1982; 69: 412.

8.- Alonso J, Barbier L, Álvarez J, et al: OK- 432 (picibanil) efficacy in an adult with cystic cervical lymphangioma. A case report. Med Oral Patol Oral Cir Bucal 2005; 10: $362-6$

9.- Rautio R, Keski-Nisula L, Laranne J, Laasonen E:
Treatment of lymphangiomas with OK-432 (Picibanil). Cardiovasc Intervent Radiol 2003; 26: 31-6.

10.- Laranne J, Keski-Nisula L, Rautio R, Rautianen M, Araksinen M: OK-432 (Picibanil) therapy for lymphangiomas in children.Eur Arch Otorhinolalyngol 2002; 259: 274

11.- Sung $M W$, Lee DW, Kim DY, et al: Sclerotherapy with picibanil (OK-432) for congenital lymphatic malformation in the head and neck. Laryngoscope 2001; 111: 1430-3.

12.- Ogita S, Tsuto T, Nakamura K, Deguchi E, Tokiwa K, Iwai $N$ : OK-432 therapy for lymphangioma in children: why and how does it work? J Pediatr Surg 1996; 31: 477-80.

13.- Greinwald JH, Burke DK, Sato Y, et al: Treatment of lymphangiomas in children: an update of Picibanil (OK432) sclerotherapy. Otolaryngol Head Neck Surg 1999; 121: 381-7.

14.- Ogita S, Tsuto T, Nakamura K, Deguchi E, Iwai N: OK432 therapy in 64 patients with lymphangioma. J Pediatr Surg 1994; 29: 784-5.

15.- Sichel JY, Udassin R, Gozal D, Koplewitz BZ, Dano I, Eliashar R: OK-432 therapy for cervical lymphangioma. Laryngoscope 2004; 114: 1805-9.

16.- Rodríguez J, Caceres F, Vargas $P$ : Treatment of Lymphangioma with OK-432 Infiltration. Cir Pediatr 2012; 25 (4): 201-4.

17.- Cuervo JL, Galli E, Eisele G, et al: Malformaciones Linfaticas: Tratamiento percutáneo con Bleomicina. Arch argent pediatr Vol 109 (5), BA sept/oct 2011.

18.- Balakrishnan K, Menezes $M$, Chen B, et al: Primary Surgery $v s$ Primary Sclerotherapy for Head and Neck Lymphatic Malformations. JAMA Otolaryn Head \& Neck Surg 2014; 140 (1): 41-5.

19.- Luzzatto C, Midrio P, Tchaprassian Z, Guglielmi M: Sclerosing treatment of lymphangiomas with OK-432. Arch Dis Child 2000; 82: 316-8.

20.- Luzzatto C, Lo Piccolo R, Fascetti León F, Zanon GF, Toffolutti T, Tregnaghi A: Further experience with OK432 for lymhangiomas. Pediatr Surger Int 2005; 21: 969-72. 